\title{
Tangence
}

\section{Les cataractes de Chassaignon}

\section{Benoît Melançon}

Numéro 57, mai 1998

Littérateurs atypiques et penseurs irréguliers

URI : https://id.erudit.org/iderudit/025969ar

DOI : https://doi.org/10.7202/025969ar

Aller au sommaire du numéro

Éditeur(s)

Tangence

ISSN

0226-9554 (imprimé)

1710-0305 (numérique)

Découvrir la revue

Citer cet article

Melançon, B. (1998). Les cataractes de Chassaignon. Tangence, (57), 72-86. https://doi.org/10.7202/025969ar d'utilisation que vous pouvez consulter en ligne.

https://apropos.erudit.org/fr/usagers/politique-dutilisation/ 


\section{Les cataractes de Chassaignon Benoît Melançon, Université de Montréal}

En 1779 paraît anonymement, en quatre volumes, probablement à Genève (IV, 372), une étrange chose intitulée Cataracies de l'imagination, déluge de la scribomanie, vomissement littéraire, hémorrbagie encyclopédique, monstre des monstres, par Épiménide l'Inspiré, Dans l'antre de Trophonius, au pays des Visions ${ }^{1}$. C'est, avec un Éloge de la Broliade par un enthousiaste, étrennes à l'auteur (1779), le coup d'envoi littéraire de Jean-Marie Chassaignon, un Lyonnais de 44 ans, qui signera par la suite des ouvrages comme Le Tiers-État rétabli pour jamais dans tous ses droits par la résurrection des bons rois et la mort éternelle des tyrans (1789) - parfois nommé Les états généraux de l'autre monde, vision propbétique -, des Étrennes à messieurs les rédacteurs du Courrier de Lyon (1790), L'offrande à Chalier, ou idées vraies et philosophiques tracées à la bâte, et offertes à son défenseur, par un homme libre et un ami des bommes (1793), Les nudités, ou les Crimes du peuple (1792) et Les ruines de Lyon (1794). Ces ouvrages ont paru entre 1789 et 1794, soit un an avant la mort de Chassaignon.

L'histoire littéraire a gardé de cet écrivain aujourd'hui obscur une image très clairement dessinée. "Mystique et instable", dit de lui le Dictionnaire des littératures française et étrangères de Larousse $^{2}$. "[L]ittérateur français, qui se fit remarquer à la fin du $\mathrm{XVIII}^{\mathrm{C}}$ siècle, par des ouvrages bizarres, produits d'un cerveau en délire", trouvait-on déjà sous la plume de J. Lamoureux dans la Nouvelle biographie générale de J.C.F. Hoefer ${ }^{3}$. Les historiens de Lyon lui font une petite place dans leurs travaux: Breghot du Lut écrivait dans ses Mélanges biographiques et littéraires pour servir

1 Lcss notes entre parenthèses renvoient à cette édition. Son orthographe et sa ponctuation ont été respectées.

2 Jacques Demougin (dir.), Dictionnaire des littératures française et étrangères, Paris, Larousse, 1992 (nouvelle édition mise à jour), xii/1861 p., p. 305.

3 Nouvelle biographie générale depuis les temps les plus reculés jusqu'à nos jours I...l, sous la direction de M. le Dr. Hoefer, Paris, Firmin Didot frères, fils et cie, 1854 , vol. 10,958 p., p. $42-43$. 
à l'bistoire de Lyon que les ouvrages de Chassaignon, "dont plusieurs sont singuliers, sont devenus fort rares et contiennent la plupart, au milieu de beaucoup de folies, des choses très-sensées et très-spirituelles " ${ }^{4}$; plus de cent cinquante ans plus tard, Georges Eynard parle de sa "véhémence verbale" et de son "abandon à la griserie des mots" 5 . Dans son Préromantisme français, André Monglond est plutôt sympathique envers Chassaignon, comme Eynard, mais il le traite néanmoins d' exalté" et de "cervelle [...] malade" ${ }^{6}$. Robert Mauzi le classe dans la section des "Imaginations morbides" de son article sur "Les maladies de l'âme au XVIII ${ }^{\mathrm{e}}$ siècle", où il côtoie Loaisel de Tréogate et Baculard d'Arnaud, et son jugement est catégorique: "Chassaignon était un malade mental." Jean Roudaut, après un article sur ce "cas littéraire", en a consacré un second à sa poésie: "Personnage hors la loi, poétique et civile, Chassaignon seul au $\mathrm{xvII}^{\mathrm{e}}$ siècle témoigne de la liberté de l'écrivain. Opposant le même refus à la vie physique (il semble avoir été en difficulté avec l'incarnation), à la vie sociale et à la vie littéraire, Chassaignon s'est refusé à écrire un livre. " ${ }^{8}$ Le portrait est unanime: instabilité, bizarrerie, véhémence, abandon, cerveau en délire, singularité, folie, exaltation, cervelle malade, malade mental, personnage hors la loi. Chassaignon est fou. Jusqu'à maintenant, dans les rares analyses de cet auteur, tout a été ramené à de l'individuel, et rien qu'à de l'individuel.

Ce portrait, Chassaignon a aidé à le dessiner. À la fin de la section "Fierté prématurée, audace, frénésie, blasphémes d'un écolier, heureuse révolution de ses idées, Vraie origine du MONSTRE LITTÉRAIRE" (IV, 270-274), on lit les deux vers suivants: "Mais où tend ma rodomontade! / On rira d'un cerveau malade"

4 Claude Breghot du lut, Mélanges biographiques et littéraires pour servir à Ibistoire de Lyon, Genève, Slatkine, 1971 (1828), 522 p., p. 402 n.

5 Georges Eynard, Joseph Chalier: bourreau ou martyr, 1747-1793, préface de Pierre Savinel, Lyon, Éditions lyonnaises d'art et d'histoire, "Collection du bicentenaite de la Révolution française à Lyon", 1987, 220 p., p. 13.

6 André Monglond, Le préromantisme français. II. Le mâtre des âmes sensibles, Paris, José Corti, 1966 (nouvelle édition), 427 p., p. 163 et p. 165.

7 Robert Mauzi, "Les maladies de l'âme au XvII ${ }^{\mathrm{e}}$ siècle", Revue des sciences bunaines, nouvelle série, 100, octobre-décembre 1960, p. 459-493, p. 489. Repris dans Maintenant sur ma route..., préface de Jean Erhard, Orléans, Paradigme, coll. "Modernités ", 1, 1994, 526 p.

8 Jean Rouclaut, "Trois poètes au début de la Révolution", Les lettres nouvelles, 3, mai 1971, p. 105-120, p. 118. Voir aussi son article intitulé "Un cas littérairc: Chassaignon", Cabiers du Sud, 346, 1957, p. 424-427. 
(IV, 272). De même, un de ses personnages parle de lui en le traitant d'uénergumène" (IV, 398). L'auteur, dont l'ironie n'était pas une vertu cardinale, était cependant conscient de ce que devait être l'image de son ouvrage aux yeux de ses contemporains et de la postérité.

S'il est incontestable, d'une part, que les Cataractes de l'imagination constituent un "ouvrage bizarre" (J. Lamoureux) et s'il est légitime, d'autre part, de croire à l'instabilité et à la mégalomanie de son auteur, on ne saurait se contenter de ramener l'entreprise de Chassaignon à la seule psychologie de celui-ci, fût-ce là une des clés de son ouvre qu'il offre lui-même à son public. Au contraire, il s'agira d'essayer de voir ce que révèle ce livre radicalement composite de l' imaginaire littéraire" à la fin du XVIII ${ }^{\mathfrak{C}}$ siècle, notamment en matière de valeurs littéraires et de mutation de la conception de la littérature ${ }^{9}$. Cela nécessite d'abord une présentation de l'ouvrage, de sa matérialité et de ce que l'on peut imaginer être son projet, afin de mettre en lumière les difficultés de lecture que posent ces Cataractes.

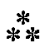

Les quatre volumes des Cataractes de l'imagination, que Chassaignon, défiant toute logique, appelle son "opuscule" (I, 6; I, 36; I, 146), se divisent, en théorie, en deux parties. La première est le "Monstre" proprement dit. Il s'agit du premier volume et d'une partie du deuxième, pour un total de 594 pages sur les 1435 que compte l'ensemble. La deuxième partie des Cataractes est intitulée "Détachement ou Entrailles du monstre" et on y regroupe des notes rattachées aux 594 premières pages. L'“ Avertissement " qui précède ces "Entrailles" expose leur nature:

Les Notes suivantes qu'annoncent les chiffres semés dans le premier \& présent volumes, y étoient d'abord consubstantiellement renfermées \& y occasionnoient une espece d'engorgement, d'obstruction, \& une bigarrure trop choquante; pour dégager la masse, vider le ventricule \& éclaircir le cahos, on a cru devoir en détacher les parties hétérogenes, indigestes \&

9) Le concept d'uimaginaire littéraire", au sens où il est utilisé ici, a été exploité par Jean M. Goulemot et Daniel Oster dans Gens de lettres, écrivains et bobèmes. L'imaginaire littéraire 1630-1900, Paris, Minerve, 1992, $199 \mathrm{p}$. 
compliquantes, \& ces notes en supplément, composent les derniers volumes (II, 232).

Cela, c'est la théorie; la pratique est un peu plus complexe.

Dans les 89 premières pages du volume initial, il y a seulement des notes infrapaginales. À partir de la p. 89, il y a trois types de notes: quelques rares notes marginales; des notes infrapaginales appelées par des lettres minuscules ou des signes typographiques; des notes reportées aux deuxième, troisième et quatrième volumes et appelées par des lettres en majuscules, puis, Z atteint, par des chiffres, de 1 à 73 . Comme si ce n'était pas suffisant, il y a, dans les notes des deuxième, troisième et quatrième volumes, de nouvelles notes infrapaginales; ce sont des notes dans les notes. Certaines notes étant fort longues - la deuxième note 59 compte 259 pages - , on comprendra que la lecture des Cataractes de l'imagination est un véritable parcours du combattant. Pour ne pas arranger les choses, après les "Entrailles du Monstre", qui font plus de deux volumes et qui se terminent par "Fin sans fin" (IV, 208), apparaît un "Arrière-monstre, plus terrible que le monstre" (IV, 209-212), puis, non moins inattendue, une "Post-face ou sequi-monstre" (IV, 275-413). Il faut attendre l'excipit pour découvrir que "Tout est consommé" (IV, 403).

Non seulement les Cataractes sont un collage de textes et de notes, et de notes dans les notes, mais les textes et les notes sont aussi des collages, puisque se mêlent à la prose de l'auteur des milliers de citations, en français, en latin, en italien, le plus souvent accompagnées d'attributions, mais pas toujours. D'une page à l'autre s'enfilent dialogues et litanies, vers et prose ${ }^{10}$. De fait, il arrive que l'on soit incapable de déterminer qui parle, cela à cause des lacunes dans la typographie (il manque souvent des guillemets).

Voilà pour la forme de l'ouvrage; qu'en est-il de son propos? de ses buts? de ses objectifs explicites? Le projet de l'auteur, si tant est qu'on puisse le définir, compense-t-il la quasi-illisibilité qu'entraîne le caractère composite de l'ensemble? La difficulté

10 Pour avoir une idée du texte, on consultera les passages qu'a retenus Michel Delon dans son Antbologie de la poésie françatse du XVIII siècle (Paris, Gallimard, coll. "Poésie", 316, 1997, 521 p., p. 245-253) ou Jacques Bousquet dans son Anthologie du dix-buitième siècle romantique (Paris, Jean-Jacques Pauvert, 1972, 574 p., p. 313-316). 
76

principale en cette matière vient de ce que Chassaignon n'expose jamais son projet synthétiquement, bien que soit postulée par lui l'existence de ce projet. Il y aurait une unité des quatre volumes, mais c'est au lecteur - il faut entendre ce mot dans un sens très large - de la reconstituer, ainsi que le révèle cette apostrophe à Dieu: "[...] Dieu vivant, qui lis dans le fond de mon ame... qui connois le but de mon ouvrage... dont le souffle brûlant pénetre j'usqu'à la moëlle de mes os... dont le sang ineffable rougit mes entrailles \& m'inonde tout entier... Être souverain, que j'invoque au lever de l'aurore, que j'invoque au coucher du soleil.. j'en appelle à toi; est-ce par une profâne animosité que j'attaque les prêtres?" (III, 17 n.) Pour qui n'est pas Dieu, la tâche de saisir le "but" de l'" ouvrage" est ardue.

Il y a plusieurs facteurs qui expliquent pourquoi il est malaisé de cerner le projet de Chassaignon. Le premier est le plus évident. Après avoir répété, dans les 400 premières pages (I, 27; I, 89-96; I, 97-143; I, 171-182), qu'il adresse un éloge au sculpteur lyonnais Poncet, ce "moderne Phidias" (II, 51), Chassaignon abandonne subitement le panégyrique au début du volume suivant :

Dieu me garde Poncet, de toucher la Donzelle;

$\mathrm{Fi}$, ta gloire est une $\mathrm{P}^{* *}$.

Qui fait coucher tout le monde avec elle.

J'y renonce: le jeu n'en vaut pas la chandelle.

[...]

Adieu, je te souhaite une bonne santé,

Dans les embrassemens de ta lubrique folle (II, 54). ${ }^{11}$

Exit Poncet, "Monsieur l'Artiste, le Philosophe, l'Académicien, \& plus que tout cela, l'ami des hommes, affable \& cher Compatriote" (I, 93-96).

À ce premier objectif délaissé s'en ajoute un deuxième qui empêche lui aussi de déceler une unité indiscutable dans l'œuvre. En effet, de la même façon que le projet initial est rapidement rejeté par Chassaignon, le retour autoréflexif de la "conclusion" - à défaut de terme plus juste - ne décrit pas la totalité du contenu explicite. Ainsi, dans "Fierté prématurée, audace, frénésie,

11 L'éloge de Chassaignon était doublement destiné à Poncet et au public: "Malgré toutes mes évolutions \& mes écarts il ne faut pas oublier que j'écris à $M$. Poncet" $(I, 127 \mathrm{n}$.); "Une fois pour toutes il ne faut point perdre de vue M. Poncet"(I, 138 n.). 
blasphémes d'un écolier, heureuse révolution de ses idées, Vraie origine du Monstre littéraire" (IV, 270-274), section qui clôt en apparence le texte, puisqu'elle est suivie de la "Post-face ou sequi-monstre" (IV, 275-413), on se voit confier l'explication du projet de l'auteur, sa "Vraie origine": on y apprend que Chassaignon s'était donné pour mission d'attaquer Voltaire et Rousseau (IV, 271). Or cette explication ne correspond qu'à certaines parties des quatre volumes ${ }^{12}$.

La rupture très nette entre les textes autoréflexifs et l'essentiel des deuxième, troisième et quatrième volumes représente une autre entrave à la saisie d'une cohérence de l'entreprise de Chassaignon. Ce qui est nettement tératologique chez lui se trouve dans les textes introductifs - "Préface qui n'en est pas une, ou échantillon de l'ouvrage" (I, 5-70) et "Avis essentiel" (I, 73-88) puis dans les derniers - "Arrière-monstre, plus terrible que le monstre" (IV, 209-212) et "Post-face ou sequi-monstre" (IV, 275413); dans les "Entrailles du Monstre", le contenu est beaucoup plus convenu. Dans le deuxième volume, par exemple, six chapitres sont consacrés à des auteurs : Jean-Baptiste Rousseau, Racine, Corneille, Boileau, Voltaire, Crébillon père, et il s'agit en l'occurrence d'évaluations, presque toujours négatives, des œuvres de ces écrivains, plus spécifiquement de leur usage du vers. Dans le troisième, on lit des passages sur Virgile, Homère, Linguet, Regnard, Théocrite, Lucain, Milton, le Tasse, Crébillon père (de nouveau), Quinault (que louange Chassaignon), suivis, dans le quatrième, de commentaires sur Pradon, Chapelain (deux fois), Scudéry, Saint-Amand, Danchet, Marmontel, Jean-Baptiste Rousseau (encore) et Fréron; dans les deux cas, Chassaignon s'emploie à montrer que les critiques ne s'entendent pas entre eux quand vient le moment de juger l'œuvre d'un auteur, qu'il soit classique ou contemporain. "Rien de si incertain que le Goût; rien de si arbitraire que le Beau" (III, 171) est le sous-titre qui coiffe cette interminable série de réflexions. Épiménide l'Inspiré s'est transformé en pion, le florilège en sottisier.

Plus ponctuellement, on note un nombre très grand de contradictions entre les premiers textes et les derniers; c'est un

12 Par exemple: "Voltaire \& Rousseau étoient des Dieux pour moi tant qu'ils vivoient; séduit par l'éclat de leur génie, étourdi du fracas de leur renommée, entraîné par l'idolâtrie générale, je m'étois jeté $\&$ couché à leurs piés. Je dormois dans ma stupide admiration!" (III, 56-57) 
quatrième facteur qui explique qu'il est laborieux de savoir où veut en venir Chassaignon dans les Cataractes de l'imagination. Au début de l'ouvrage, il chante la liberté du créateur, il vante les charmes de la transe:

Nos grammairiens, nos puristes \& nos rhéteurs auront beau, leur Quintilien, leur Batteux, \& leur Vailli en main, faire le procès à mes écarts \& à mes incorrections; d'un seul éclat de mon imagination je foudroierai ce pusillanime troupeau d'esclaves nés pour aligner des mots, symétriser des phrases, \& couper les ailes du génie.

Un littérateur dont l'ame est brulante, \& le cerveau exalté, doit, dans la fougue de son délire, être incapable de mettre certaine suite dans ses conceptions, certaine harmonie dans ses discours; comme il ne pense que par secousse, comme il n'écrit que par inspiration, quand il a versé sur le papier l'idée qui l'obsédoit, il ne doit plus se rappeler de ce qu'il a pensé, il ne doit plus savoir ce qu'il va écrire (I, 15-16).

Après pareille affirmation, on s'étonne de voir Chassaignon défendre l'ordre et le bon goût dans la suite de son ouvrage, on le voit mal reprocher à Crébillon père son manque de "simplicité noble et touchante" (II, 141), on ne saisit pas pourquoi il est nécessaire de respecter la "majesté de l'ode" (IV, 70). De même, on ne s'attendait pas à apprendre que Chassaignon n'aime pas Milton parce que Paradise Lost est une "production irrégulière \& étrange" (III, 243). L'auteur, qui n'est pourtant pas homme à reculer devant la contradiction, essaie de s'innocenter par avance des accusations qu'on pourrait lui adresser à cet égard: "Qu'on ne cherche point ici à me mettre en contradiction avec moi-même, en m'opposant le radotage combiné du "Monstre". J'ai déja averti que les mistérieuses extravagances dont-il est tissu [sic], ne doivent point être prises à la lettre (IV, $323 \mathrm{n}$.). Voilà une prescription de lecture qui dédouane à bon compte.

Parce qu'il ne réalise ni son apparent projet initial ni celui qu'il récapitule en conclusion, parce qu'il est considérablement plus sage que pourraient le laisser croire ses textes programmatiques et parce qu'il se contredit, Chassaignon livre bel et bien un objet totalement hétéroclite, d'un "genre inconnu à ce siècle" (I, 6) et propre à "épouvanter le public" (I, $10 \mathrm{n}$.). 
Quiconque se limiterait à constater la bigarrure de l'œuvre, les incohérences de son contenu et les problèmes de lecture qu'elle pose aurait tôt fait de classer Chassaignon au rang des fous littéraires, et c'est, pour l'essentiel, ce que la critique a cru jusqu'à ce jour. Pour elle, Jean-Marie Chassaignon ne serait qu'un cas isolé, peut-être splendidement. Pareille catégorisation - qui n'est évidemment pas sans fondement - a pour conséquence principale de renvoyer les Cataractes de l'imagination dans un hors-lieu discursif, comme si Chassaignon, parce que fou, n'avait rien à révéler de l'état du discours à la veille de la Révolution. Or on peut postuler, au contraire, qu'il a quelque chose à dire sur l'axiologie littéraire de son temps et sur les prémices de la modernité.

Dans un premier temps, il faut souligner que Chassaignon a beau être atypique, mais qu'il n'en est pas moins homme des Lumières, et il ne paraît donc pas qu'il faille suivre Fritz-Günther Frank lorsqu'il le traite d' antiphilosophe" dans sa thèse ${ }^{13}$. De fait, Chassaignon partage avec ceux que l'on appelle les philosophes bon nombre de valeurs: le "vrai désir d'être utile" (I, 38), la haine des préjugés (I, 203; IV, 7; IV, 395), le refus du fanatisme (II, 309s), le relativisme des mœurs - il se réclame de Montaigne, qui est un des rares auteurs à trouver fréquemment grâce à ses yeux -, l'importance de la propriété littéraire - il appuie Luneau de Boisjermain dans un procès devenu célèbre ${ }^{14}$ (I, 180 n.) -, la défense de la morale (IV, 395), l'éloge du génie (I, 193-213), l'amour de la vertu (IV, 192-193; IV, 256 n.) et l'apologie des passions fortes - quand il écrit "Les folies \& les crimes immortalisent comme les plus belles actions" (I, 186), on croit entendre le Diderot du Neveu de Rameau ${ }^{15}$. On pourrait certes énumérer les valeurs qui distinguent Chassaignon: la religion, sinon le mysticisme, le pouvoir monarchique sans contre-pouvoir (III, 98), le refus du roman et des nouvelles formes théâtrales (II,

13 Fritz-Günther Frank, "Jean-Marie Chassaignon: ein Antiphilosoph zwischen klassizistischer Tradition und Moderner Ästhetik", Stuttgart, Université de Stuttgart, thèse de doctorat, 1973, 176 p. Voir le compte rendu dans Frencb Studies, 31, 3, juillet 1977, p. 333-334.

14 Voir John Lough, "Luneau de Boisjermain v. the Publishers of the Encyclopédie", Studies on Voltaire and the Eighteenth Century, 23, 1963, p. 115-177. Repris dans The Encyclopédie in Eigbteentb Century England and Other Studies, Newcastle upon Tyne, Oriel Press, 1970 , viii/256 p., p. 96-158.

15 Voir Diderot, Le neveu de Rameau, édition critique avec notes et lexique par Jean Fabre, Genève, Droz, coll. “Textes littéraires français ", 37, 1977 (1950), $\mathrm{xcv} / 329$ p., p. $72-76$. 
80

262-264), la résistance au rationalisme ${ }^{16}$, et on pourrait discuter des définitions de Chassaignon des valeurs qu'il prône - ces définitions ne sont peut-être pas toujours celles de ses contemporains -, mais la seule présence de ces valeurs empêche de le reléguer dans les marges des Lumières.

Semblablement, Chassaignon rejoint un écrivain aussi fortement légitimé que Rousseau dans la revendication d'une valeur nouvelle à la fin de l'Ancien Régime, l'individualité. Conscient de son "originalité" $(I, 6)$ et de sa "singularité" $(I, 7)$, ainsi que de la "solitude" qu'elles entraînent (I, 222-229), il a des passages dignes des Confessions - mais qui précèdent leur publication. Tel extrait du premier volume, où apparaît nommément "J. Jacques", doit en effet être lu en parallèle avec l'incipit de l'autobiographie de Rousseau.

Voltaire, J. Jacques, Corneille ni Montesquieu n'ont pas senti ce que je sens. Je préfere moi à tous ces fastidieux personnages. Je préfere moi à tout ce qui existe; c'est avec ce moi seul que j'ai passé les plus doux momens de ma vie; ce moi isolé, entouré de tombeaux, \& invoquant le grand être, suffiroit à mon bonheur sur les décombres de l'univers... La perfidie d'un ami m'eût fait moins de peine, que son importunité, lorsqu'il est venu m'arracher à moi-même... dans les rues où je me plais à marcher seul, je suis dans la crise d'un homme égaré dans un bois rempli d'assassins ou de bêtes féroces: le moindre objet m'allarme; l'éclair de mon œil va saisir le regard du premier qui me coudoie: s'il m'envisage, je recule; c'est un attentat qu'il médite; il en veut à la jouissance de moi-même; il ne m'aborde que pour me nuire; il va supplanter en me parlant, le génie avec lequel je converse; \& dont son entretien ne peut me dédommager... que ne suis-je transporté dans une contrée barbare où personne ne me connoisse, où je ne sois interrompu par aucun ami, où moi m'appartienne tout entier; une demi-. heure qu'on m'enleve, est une verrée de sang qu'on me tire, est un lambeau de mon cour qu'on m'arrache (I, 79-81).

Culte de l'individualité, éloge de la solitude, affirmation de la nouveauté de ce que le lecteur va découvrir, évocation de la mort et du tribunal céleste: sans prétendre faire de Chassaignon un précurseur de Rousseau, ou son égal au panthéon des inventeurs

16 Selon Andrew Curran et Patrick Graille, les Cataractes " is expressly designed to counter the Enlightenment's attempt to advance a rational language and correspondingly rational world" "The Faces of Eighteenth-Century Monstrosity ", Eighteenth-Century Life, 21, n.s., 2, mai 1997, p. 1-15, p. 9). 
de formes, il importe de signaler ces analogies, de proposer des rapprochements ${ }^{17}$.

A ce type d'inscription des Cataractes de l'imagination dans l'imaginaire littéraire des Lumières tardives - Jean-Marie Chassaignon est homme des Lumières et de leurs contradictions -, un deuxième s'ajoute, nettement plus fécond. À titre d'hypothèse, on pourrait formuler les choses ainsi : l'ouvrage de Chassaignon est un signe, et un signe particulièrement éloquent, d'une autonomisation du champ littéraire dès le troisième quart du siècle des Lumières (pour parler en termes bourdieusiens), d'une clôture de l'imprimé proche de celle que viendra à symboliser la notion de modernité ${ }^{18}$. Ce marginal parle du cœur des Lettres, il les a pour objet et pour matériau, il se nourrit de mots, et rien que d'eux. Plus radicale que celle de Rousseau à cet égard, son œuvre refuse ostensiblement la parole publique: on peut imaginer lire les Confessions devant une assemblée, par exemple dans un salon, mais pas les Cataractes. Encore une fois, il ne s'agit pas de faire de Chassaignon un grand précurseur oublié, mais de réfléchir à partir de lui à un phénomène qu'on a accoutumé de situer plus loin dans le temps, soit la clôture du texte sur lui-même. Cela prend chez Chassaignon des formes multiples.

Sur le plan thématique, les Cataractes de l'imagination carburent presque uniquement à la littérature. Adversaire patenté de celle-ci ou, plus justement, de la "versimanie" (I, 108), l'auteur du Monstre écrit comme s'il n'avait pas la possibilité d'aborder d'autre sujet: "Disons puisqu'il le faut du mal des poëtes" (II, 68), lance-t-il en incipit du chapitre XXVIII du $2^{\mathrm{e}}$ volume. Ce refus affiché de la littérature se nourrit pourtant des œuvres littéraires, comme l'indique telle autojustification:

17 La question des rapports entre Chassaignon et Rousseau est d'autant plus complexe que les Cataractes ont paru avant les Confessions, mais que Rousseau a lu des passages de celles-ci avant leur publication et avant la parution de l'ouvrage de Chassaignon. On mettra également en parallèle cet extrait de Chassaignon avec la troisième des lettres autobiographiques de Rousseau à Malesherbes, celle du 26 janvier 1762, qui sera publiêe la même année que les Cataractes (Guvres complètes. I. Les confessions. Autres textes autobiographiques, édition publiée sous la direction de Bemard Gagnebin et Marcel Raymond, Paris, Gallimard, coll. "Bibliothèque de la Pléiade ", 11, 1996 [1959], cxviii/1969 p., p. 1138-1141).

18 Vór, par exemple, Antoine Compagnon, Les cinq paradoxes de la modemité, Paris, Seuil, 1990, 189 p., p. 36. 
82

Ai-je assassiné mon pere? En ai-je profané les cendres? Ai-je souillé quelque sanctuaire? Suis-je le meurtrier des rois, l'empoisonneur des papes? Condamnez-moi plutôt à manger de l'ail, infernal aliment qu'Horace destinoit aux paricides; du pain fait avec des os de mort pulvérisés, comme en 1590, les Parisiens furent réduits à s'en nourrir, lors du Blocus de la ville: donnez-moi pour breuvage, du sang corrompu de taureau, comme Pelias, le plus cruel des hommes, en faisoit prendre à Eson, dont il avait envahi les états, \& qu'il tenoit renfermé dans un cachot. Voulez-vous mieux, tenez je me résigne à lire un quart d'un chant de la Pucelle de Chapelain, à dévorer tout l'article chapeau de l'encyclopédie, à éssuyer la cruelle longueur d'une these sorbonique, à feuilleter périodiquement l'éthique et fastidieux mercure de France, à supporter constamment toutes les assommantes représentations de nos drames bourgeois, \& fussiez-vous plus acharné contre moi, que le roi Euristhée le fut contre le fils d'Alcmene, auquel il suscita tant de travaux, plus inexorable que la reine des dieux qui voua aux Troyens une haine si terrible, je viendrai peut-être à bout de vous calmer en m'engageant à parcourir pendant dix années, si le dégoût \& l'ennui n'abrégent point auparavant mes jours, les quatre-vingt \& dix énormes \& sempiternels volumes de complimens que l'académie françoise a recueillis. [...] Moi poëte! j'irai crucifier mon cerveau à pure perte! (I, 104-107)

$\mathrm{Ni}$ assassin, ni profanateur, ni régicide, ni empoisonneur, ni poète, Chassaignon reste un lecteur impénitent.

On vient de le voir: ce lecteur ne loupe pas une occasion d'étaler son érudition. Si elle est parfois historique, comme dans ce catalogue de sévices, elle se caractérise également par le fait que Chassaignon chérit les écrits qui ont la littérature pour sujet. S'il lit Piron, c'est La métromanie ou le Poète (1738). S'il parcourt Alexander Pope, c'est La Dunciade (1728 et 1742) ou L'art de ramper en poésie (Peri Batbous, or the Art of Sinking in Poetry, 1727, publié sous le pseudonyme de Martinus Scriblerus). S'il consulte des journaux, ce sont des gazettes littéraires, le Mercure, le Journal politique et littéraire de Linguet, L'année littéraire de Fréron, des Annales. Complètement étrangère au ludisme ou à la parodie dont Thémiseul de Saint-Hyacinthe témoigne dans son Cbef-d'œuvre d'un inconnu (1714) ${ }^{19}$, son érudition est sans cesse

19 Voir la contribution d'Éric Méchoulan, que rejoint à plusieurs égards la présente lecture de Chassaignon, notamment en ce qui concerne la transition de l'oral à l'écrit au XvıII" siècle. 
martelée: jamais on ne saurait oublier qu'on a sous les yeux le fruit du travail d'un esprit encyclopédique. Se déploient à chaque page ses techniques de prédilection: le collage, l'accumulation, la concaténation, la couture (la métaphore est récurrente), la marqueterie, le catalogue, la convocation d'autorités de toutes les époques et de tous les temps - en un mot, l'" hémorrhagie encyclopédique" du titre. Chassaignon ne cesse d'aligner ou d'imbriquer, au risque du vertige, des citations, des anecdotes, des paraphrases, des "traits" d'histoire (I, 340). Il prend son bien où celui-ci l'attendait: "Pour achever ma préface, j'y vais coudre une partie de celle des pensées errantes de Diderot qu'il a faite exprès pour moi" (I, 68). Cela donne des pages où toutes les phrases commencent par "Ainsi" (I, 131-137; I, 140-141; I, 316-318) ou par "Comme" (I, 104-107; II, 294-295), des chapitres composés presque exclusivement de mots et d'idées empruntés (II, ch. XIX, XXII et XXX; III, "Le lapon ou le cuisinier d'Alger"), des passages où l'on ne sait pas qui parle, tellement les emprunts sont nombreux. La tâche de Chassaignon est colossale et il ne s'en cache pas:

Une compilation ordinaire n'est pas, j'en conviens, d'une difficulté bien étrange; mais quand, dans le cours d'un long ouvrage, on s'est imposé la loi d'investir chaque pensée d'un escadron de traits d'histoires, d'épigraphes, d'apophtegmes puisés dans des auteurs différens, la besogne alors est bien pénible; combien, pour remplir une seule page, ne faut-il pas avoir dévoré de volumes? (I, $37 \mathrm{n}$.)

Qui aurait craint que la boulimie lectoriale de Chassaignon ne devienne la source d'une aboulie créatrice se serait gravement trompé : cette boulimie est la condition de sa pratique littéraire.

Une des figures fondamentales du texte est la circulation pour le dire en termes volontairement très généraux et polis des fluides et des solides dans et à l'extérieur des corps. Il n'y a pas que l'eau qui tombe des cataractes; Chassaignon a une fascination très nette pour l'ingestion, l'allaitement, la digestion, le vomissement (voir le titre), l'expectoration, l'éternuement, la miction (I, 307 n.), la défécation (II, 143), le cannibalisme, etc. Certaines anecdotes, si elles n'étaient pas relayées par ce réseau métaphorique, pourraient n'être que cocasses. "On dit que Jupiter enfant étant dans l'isle de Crête où Rhée sa mere l'avoit donné à élever aux Coribantes, le géant Typhont s'élança sur lui \& le dévora; mais comme les Dieux sont durs à digérer, le monstre fut 
obligé de le revomir, \& Jupiter, quoique trituré, reprit sa premiere forme" (I, 206 n.); ce microrécit mythologique s'inscrit dans un vaste ensemble où corps et création sont intimement fondus. Le littéraire n'échappe pas à ce commerce généralisé des matières.

Je compare un auteur novice qui s'essaie, à un homme enrhumé à qui les narines démangent, \& qui se tourmente pour éternuer; si vous le brusquez par une secousse violente, si vous le surprenez par un éclat de voix trop fort, il s'arrête sans pouvoir consommer l'acte qu'il commençoit; l'éternument va toujours venir, \& ne vient jamais; si au contraire vous facilitez les efforts par quelque poudre bénignement irritante, l'éternuement se décide, l'humeur coule, \& le cerveau est soulagé; il en est de même à l'égard du génie en travail ; l'aboiement de la satyre le fait avorter, tandis qu'une vapeur d'encens eût au contraire aidé son accouchement (II, 30).

L'activité littéraire, telle que la conçoit Chassaignon, est affaire de conversion et de dissémination, d'ingestion et de digestion. Or, comme on l'a vu, ce qui circule dans ses quatre volumes, c'est du textuel. Son monstre littéraire est fait de la somme (considérable) de ses lectures et de leur métamorphose.

Quand il n'est pas à digérer des écrits divers, l'auteur des Cataractes polémique tous azimuts. Non seulement il en profite pour marquer son territoire dans l'univers des discours de son époque et se doter d'un capital symbolique au détriment de ceux qui l'entourent, mais il rappelle encore par là qu'il est lui-même fait de mots. Il n'épargne aucun de ses concitoyens de la "France littéraire" (I, 159 n.), de la "contrée littéraire" (I, 283), du "territoire poétique" (I, 298) ou de "l'empire littéraire" (II, 330). Il a ses têtes de Turc, Boileau ou le "trop célebre Linguet" (II, 242) et ses "beuglements sempiternels" (IV, 52), mais presque tous ses contemporains y passent, tenants qu'ils sont d'un pouvoir dont ils lui bloquent l'accès (ce sont les "Cerberes de la littérature", I, 185). Il va dès lors s'en prendre à Marmontel, à La Harpe, aux deux Fréron, à ceux qu'il appelle les "géomètres" (passim), aux membres de l'Académie française et à la "fourmiliere d'insectes académisés" (IV, 346), au "vatican philosophique" (I, 66 n.) et à "l'inquisition du Parnasse" (II, 125), aux "tyrans" (I, 148) comme aux " princes littéraires" (II, 203), à ceux qui occupent le "parlement littéraire" (I, 262) ou qui sont installés sur le "siège soporifique" (I, 266). Il aura aussi quelques bons mots - pourquoi s'en priver? - pour la "lie du peuple littéraire" (I, 298) et sa "cannaille" (I, 
343). Armé du "poignard littéraire" (I, 249), ce législateur propose des lois contre l'application au "vulgaire des expressions consacrées à l'éloge des Dieux, des héros \& des Rois" (II, 104) et il suggère d'instituer une "taxe pour détruire l'incommode engeance de ces rimailleurs impitoyables, qui nous poursuivent dans tous les coins de rue, \& attentent sans cesse à notre repos" (II, 306-307). Autoproclamé juge, il dresse son propre "tribunal", auquel il convoque Boileau (II, 145). Au début de la section "Paraphrase des prophéties d'Ézéchiel, \&c. \&c. Visions, enfers, apocalypse nouvelle. Offrande au clergé. Dialogue en action entre l'Auteur du Monstre \& son Génie" (p. 213-274), ledit Génie lui accorde les pleins pouvoirs: "De l'univers je t'établis l'arbitre" (IV, 214). Ce "nouveau dictateur de la république des lettres" (I, 35) mène une guerre sainte qui est une guerre de littérature.

La thématique, l'érudition revendiquée, les métaphores de la circulation des fluides et des solides, et la polémique sont autant de formes que prend la clôture du littéraire dans les Cataractes de Chassaignon; il en va de même du nouveau rôle que s'y confère l'auteur. Dans Le sacre de l'écrivain, Paul Bénichou l'avait bien vu, qui prenait Chassaignon pour exemple, mais caricatural, d'une transformation en cours, dans les années qui précèdent la Révolution, du statut de l'écrivain. La "poétique religieuse plus large" dont Bénichou suit la lente naissance au cours du "XVIII siècle chrétien", "l'intuition ou le désir d'une refonte des valeurs littéraires" et la mutation d' "une tradition ancienne [...] à l'appel d'un besoin nouveau" seraient actifs chez Chassaignon, " préromantique effréné, catholique aberrant, contre-révolutionnaire et demi-fou"; "le Poète" s'y ferait "Messie pour exercer un ministère actuel dans une sorte de jugement dernier des philosophes mécréant " ${ }^{20}$. Au fur et à mesure qu'avance le texte, les figures de la vision, la religion et la prophétie occupent une place croissante. Écrivant "Mon ceil, de l'avenir, a percé l'enveloppe..." (IV, 231) ou "Au choc du Monstre l'ennemi littéraire va éprouver une secousse... une révolution..." (IV, 402), Chassaignon devient un mage et il n'est plus un créateur comme les autres. Ayant traversé le littéraire, il s'investit d'une mission: dévoiler ce qui est caché, annoncer ce qui vient. À défaut d'un talent de

20) Paul Bénichou, Le sacre de l'écrivain. 1750-1830. Essai sur l'avènement d'un pouvoir spirituel läque dans la France moderne, Paris, Gallimard, coll. " Bibliothèque des idées n, 1996 (1973), 492 p., p. 88. 
86

pronostiqueur, on lui reconnaîtra la conscience, ne serait-ce que partielle, d'une nouveauté: "Si je ne suis pas heureux, j'aurai du moins été hardi" (I, 186).

Même si, à en croire Voltaire dans la troisième de ses Lettres philosophiques, "l'enthousiasme est une maladie qui se gagne" 21 , Chassaignon n'a pas eu de sectateurs. Longtemps oublié, il s'inscrit pourtant parmi ces minores et ces critiques des Lumières dont l'étude peut être utile à la compréhension du monde des lettres au $\mathrm{XVIII}^{\mathrm{e}}$ siècle $^{22}$. Sans vouloir le réhabiliter, ainsi que le tentait Jean Roudaut en $1957^{23}$, on a cherché ici à montrer comment une œuvre en apparence aussi atypique et marginale que la sienne révèle les modifications de l'imaginaire littéraire de son époque. Tant sur le plan de l'axiologie que de la conception du littéraire en 1779 , les Cataractes de l'imagination sont l'œuvre d'un homme des Lumière. Isolé, certes, bizarre, indubitablement, ce texte n'est pas à l'abri des discours qui l'entourent et le nourrissent, des valeurs qui se disputent la prééminence dans le monde des belles-lettres, des conditions d'apparition de cette chose nouvelle au XVIII ${ }^{e}$ siècle, la littérature.

21 Voltaire, Lettres pbilosophiques, chronologie et préface par René Pomeau, Paris, GF-Flammarion, coll, "GF", 15, 1964, 188 p., p. 31.

22 Sur le rôle que jouent les minores dans le renouveau des études clix-huitićmistes, voir Michel Delon, "Quelques remarques sur les objets de l'histoire littéraire en France aujourd'hui ", dans Revue d'bistoire littéraire de la France. Collogue du centenaire, Paris, Armand Colin, Société d'histoire littéraire de la France, 1995 , p. 171-175, p. 173. Sur les critiques des Lumières, on lira, du même, "Les Lumières aujourd'hui: l'universel et le particulier", Studies on Voltaire and the Eighteenth Century, 346, 1996, p. 163-171: "Notre attention se porte sur la relation entre Lumières et non-Lumières, c'est-à-dire non seulement les Anti-Lumières constituées en groupe ou en front contre le clan encyclopédique, mais aussi les marges des Lumières et toutes les résistances singulières qu'elles ont suscitées" (p. 165). Les minores pornographiques sont l'objet d'un article de Jean $M$. Goulemot, "Minores et livres de second rayon du XVIII "siècle", Littératures classiques, 31, 1997, p. 135-144.

23 Il parlait de "déni de justice" (Un cas littéraire: Chassaignon", loc. cit., p. 424). 